\title{
TENSORS AND DIFFERENTIAL FORMS
}

\author{
SVANTE JANSON \\ UPPSALA UNIVERSITY
}

\section{INTRODUCTION}

The purpose of these notes is to give a quick course on tensors in general differentiable manifolds, as a complement to standard textbooks.

Most proofs are quite straightforward, and are left as exercises to the reader.

The remarks contain additional information, but may be skipped by those so inclined.

More details can be found in, for example, Boothby [1] and Warner [3].

\section{TENSORS OVER A VECTOR SPACE}

Throughout this section, $V$ is a finite-dimensional real vector space. The dimension $\operatorname{dim}(V)$ will be denoted by $n$.

Remark. It is also possible to define tensors over a complex vector space (with no essential modifications in the definitions and results below, except that the special Euclidean case no longer applies). This is important in many other applications (including complex manifolds), but will not be considered here.

1.1. The dual space. Let $V^{*}=\operatorname{hom}(V ; \mathbb{R})$ be the dual of $V$, i.e. the vector space of all linear functionals $V \rightarrow \mathbb{R}$. We write $\left\langle v^{*}, v\right\rangle$ for $v^{*}(v)$, where $v^{*} \in V^{*}$ and $v \in V$.

Note that $V$ and $V^{*}$ are isomorphic vector spaces, since they have the same dimension, but there is, in general, no specific natural isomorphism between them and it is important to distinguish between them. (For the Euclidean case, see below.) On the other hand, there is a natural isomorphism $V \cong V^{* *}$ between $V$ and its second dual, and these space may be identified. ( $v \in V$ corresponds to $v^{* *} \in V^{* *}$ given by $\left\langle v^{* *}, v^{*}\right\rangle=\left\langle v^{*}, v\right\rangle, v^{*} \in V^{*}$.)

If $\left\{e_{1}, \ldots, e_{n}\right\}$ is a basis in $V$, so that the elements of $V$ may uniquely be written $\sum_{1}^{n} a^{i} e_{i}, a^{i} \in \mathbb{R}$, we define $e^{1}, \ldots, e^{n} \in V^{*}$ by

$$
e^{i}\left(\sum_{1}^{n} a^{j} e_{j}\right)=a^{i} .
$$

It is easily seen that $\left\{e^{1}, \ldots, e^{n}\right\}$ is a basis in $V^{*}$, called the dual basis of $\left\{e_{1}, \ldots, e_{n}\right\}$. We have

$$
\left\langle e^{i}, e_{j}\right\rangle=\delta_{i j}= \begin{cases}1, & i=j \\ 0, & i \neq j .\end{cases}
$$

Date: April 17, 2000; corrected April 27, 2000; typo corrected April 17, 2011. 
In other words, the matrix $\left(\left\langle e^{i}, e_{j}\right\rangle\right)_{i, j=1}^{n}$ is the identity matrix.

Note that duality of bases is a symmetric relation; using the identification of $V^{* *}$ and $V$, the dual basis of $\left\{e^{1}, \ldots, e^{n}\right\}$ is $\left\{e_{1}, \ldots, e_{n}\right\}$.

If $\left\{f_{1}, \ldots, f_{n}\right\}$ is another basis in $V$, then $f_{i}=\sum_{j} a_{i j} e_{j}$ for some numbers $a_{i j}$, and similarly for the corresponding dual bases $f^{i}=\sum_{j} b_{i j} e^{j}$. We have $\left\langle f^{i}, f_{j}\right\rangle=\sum_{k} b_{i k}\left\langle e^{k}, f_{j}\right\rangle=\sum_{k} b_{i k} a_{j k}$, and thus, introducing the matrices $A=$ $\left(a_{i j}\right)_{i j}$ and $B=\left(b_{i j}\right)_{i j}$,

$$
I=\left(\left\langle f^{i}, f_{j}\right\rangle\right)_{i j}=B A^{t} .
$$

Consequently, the matrices of the changes of bases are related by $B=\left(A^{t}\right)^{-1}=$ $\left(A^{-1}\right)^{t}$.

1.2. Tensors. For two non-negative integers $k$ and $l, T^{k, l}(V)$ is defined to be the vector space of all multilinear maps $f\left(v_{1}^{*}, \ldots, v_{k}^{*}, v_{1}, \ldots, v_{l}\right): V^{*} \times \cdots \times$ $V^{*} \times V \times \cdots \times V \rightarrow \mathbb{R}$, with $k$ arguments in $V^{*}$ and $l$ arguments in $V$. The elements of $T^{k, l}(V)$ are called tensors of degree (or order) $(k, l)$.

Given a basis $\left\{e_{1}, \ldots, e_{n}\right\}$ of $V$, a tensor $T$ of degree $(k, l)$ is uniquely determined by the $n^{k+l}$ numbers (which we call coefficients of the tensor)

$$
T_{j_{1}, \ldots, j_{l}}^{i_{1}, \ldots, i_{k}}=T\left(e^{i_{1}}, \ldots, e^{i_{k}}, e_{j_{1}}, \ldots, e_{j_{l}}\right) .
$$

Conversely, there is such a tensor of degree $(k, l)$ for any choice of these numbers. Thus the vector space $T^{k, l}(V)$ has dimension $n^{k+l}$.

A tensor of type $(k, 0)$ for some $k \geq 1$ is called contravariant and a tensor of type $(0, l)$ for some $l \geq 1$ is called covariant. Similarly, in the coefficients (1.1), the indices $i_{1}, \ldots, i_{k}$ are called contravariant, and the indices $j_{1}, \ldots, j_{l}$ are called covariant. It is conventional, and convenient, to use superscripts for contravariant indices and subscripts for covariant indices.

Example 1.2.1. The case $k=l=0$ is rather trivial (but useful); $T^{0,0}(V)$ is just the vector space of all real numbers, $T^{0,0}(V)=\mathbb{R}$ and a tensor of degree $(0,0)$ is a real number. Tensors of degree $(0,0)$ are also called scalars.

Example 1.2.2. $T^{0,1}(V)$ is the space of all linear maps $V \rightarrow \mathbb{R}$. Thus $T^{0,1}(V)=V^{*}$, and a tensor of degree $(0,1)$ is a linear functional on $V$.

Example 1.2.3. Similarly, $T^{1,0}(V)$ equals $V^{* *}$, which we identify with $V$. Thus, $T^{1,0}(V)=V$, and a tensor of degree $(1,0)$ is an element of $V$.

Example 1.2.4. A linear mapping $S: V \rightarrow V$ defines a tensor $T$ of degree $(1,1)$ by

$$
T\left(v^{*}, v\right)=\left\langle v^{*}, S(v)\right\rangle ; \quad v^{*} \in V^{*}, v \in V .
$$

In a basis $\left\{e_{1}, \ldots, e_{n}\right\}$, this tensor has the coefficients

$$
T_{j}^{i}=T\left(e^{i}, e_{j}\right)=\left\langle e^{i}, S\left(e_{j}\right)\right\rangle=s_{i j},
$$

where $s_{i j}$ is the matrix representation of $S$ in the basis $\left\{e_{1}, \ldots, e_{n}\right\}$. Hence (1.2) defines a natural bijection between $T^{1,1}(V)$ and the space hom $(V ; V)$ of all linear mappings $V \rightarrow V$, so we may identify $T^{1,1}(V)$ and $\operatorname{hom}(V ; V)$ and regard a tensor of degree $(1,1)$ as a linear mapping $V \rightarrow V$. Note that the identity mapping $V \rightarrow V$ thus corresponds to the special tensor $\iota \in T^{1,1}(V)$ 
given by $\iota\left(v^{*}, v\right)=\left\langle v^{*}, v\right\rangle$, with coefficients $\iota_{j}^{i}=\delta_{i j}$ (in this context better written $\left.\delta_{j}^{i}\right)$.

Example 1.2.5. More generally, a multilinear mapping $S: V^{l} \rightarrow V$ defines a tensor $T$ of degree $(1, l)$ by $T\left(v^{*}, v_{1}, \ldots, v_{l}\right)=\left\langle v^{*}, S\left(v_{1}, \ldots, v_{l}\right)\right\rangle$. Hence we may identify $T^{1, l}(V)$ and the $\operatorname{space} \operatorname{hom}(V, \ldots, V ; V)$ of all such multilinear mappings.

1.3. Tensor product. Tensors may be multiplied by real numbers, and two tensors of the same degree may be added, because each $T^{k, l}(V)$ is a vector space. Moreover, there is a multiplication, known as tensor product such that any two tensors may be multiplied. The tensor product of two tensors $T$ and $U$ of degrees $\left(k_{1}, l_{1}\right)$ and $\left(k_{2}, l_{2}\right)$, respectively, is a tensor of degree $\left(k_{1}+k_{2}, l_{1}+l_{2}\right)$ denoted by $T \otimes U$ and defined by

$$
\begin{aligned}
& T \otimes U \\
& \quad\left(v_{1}^{*}, \ldots, v_{k_{1}+k_{2}}^{*}, v_{1}, \ldots, v_{l_{1}+l_{2}}\right) \\
& \quad=T\left(v_{1}^{*}, \ldots, v_{k_{1}}^{*}, v_{1}, \ldots, v_{l_{1}}\right) U\left(v_{k_{1}+1}^{*}, \ldots, v_{k_{1}+k_{2}}^{*}, v_{l_{1}+1}, \ldots, v_{l_{1}+l_{2}}\right) .
\end{aligned}
$$

If $T$ or $U$ has order $(0,0)$, the tensor product reduces to the ordinary multiplication of a tensor by a real number.

By (1.1) and (1.3), the coefficients of the tensor product are given by

$$
(T \otimes U)_{v_{1}, \ldots, v_{l_{1}+l_{2}}^{*}}^{v_{1}^{*}, \ldots, v_{k_{1}}^{*}+k_{2}}=T_{v_{1}, \ldots, v_{l_{1}}}^{v_{1}^{*}, \ldots, v_{k_{1}}^{*}} U_{v_{l_{1}+1}, \ldots, v_{l_{1}+l_{2}}}^{v_{k_{1}}^{*}+T_{1}, \ldots, v_{1}^{*}}
$$

The tensor product is associative; $T_{1} \otimes\left(T_{2} \otimes T_{3}\right)=\left(T_{1} \otimes T_{2}\right) \otimes T_{3}$ for any three tensors $T_{1}, T_{2}, T_{3}$, so we may write $T_{1} \otimes T_{2} \otimes T_{3}$ etc. without any danger for the tensor product of three or more tensors. The tensor product is not commutative, however, so it is important to keep track of the order of the tensors. The tensor product is further distributive; $\left(T_{1}+T_{2}\right) \otimes T_{3}=$ $T_{1} \otimes T_{3}+T_{1} \otimes T_{3}$ and $T_{1} \otimes\left(T_{2}+T_{3}\right)=T_{1} \otimes T_{2}+T_{1} \otimes T_{3}$.

Remark. The tensor algebra is defined as the direct sum $\bigoplus_{k, l=0}^{\infty} T^{k, l}(V)$ of all the spaces $T^{k, l}(V)$; its elements are thus sums of finitely many tensors of different degrees. Any two elements in the tensor algebra may be added or multiplied (so it is an algebra in the algebraic sense).

We will not use this construct, which sometimes is convenient; for our purposes it is better to consider the spaces $T^{k, l}(V)$ separately.

Remark. There is also a general construction of the tensor product of two vector spaces. We will not define it here, but remark that using it, $T^{k, l}(V)=$ $V \otimes \cdots \otimes V \otimes V^{*} \otimes \cdots \otimes V^{*}$ (with $k$ factors $V$ and $l$ factors $V^{*}$ ), which yields another, and perhaps more natural, definition of the tensor spaces $T^{k, l}(V)$.

1.4. Bases. If $\left\{e_{1}, \ldots, e_{n}\right\}$ is a basis in $V$, we can for any indices $i_{1}, \ldots, i_{k}$, $j_{1}, \ldots, j_{l} \in\{1, \ldots, n\}$ for the tensor product

$$
e_{i_{1}} \otimes \ldots \otimes e_{i_{k}} \otimes e^{j_{1}} \otimes \ldots \otimes e^{j_{l}} \in T^{k, l}(V) .
$$

(Recall that $e_{i} \in T^{1,0}(V)$ and $e^{j} \in T^{0,1}(V)$.) It follows from (1.1) and (1.3) that this tensor has coefficients

$$
e_{i_{1}} \otimes \ldots \otimes e_{i_{k}} \otimes e^{j_{1}} \otimes \ldots \otimes e^{j_{l}}\left(e^{i_{1}^{\prime}}, \ldots, e^{i_{k}^{\prime}}, e_{j_{1}^{\prime}}, \ldots, e_{j_{l}^{\prime}}\right)=\delta_{i_{1} i_{1}^{\prime}} \delta_{i_{2} i_{2}^{\prime}} \ldots \delta_{j_{l} j_{l}^{\prime}}
$$


in the basis $\left\{e_{1}, \ldots, e_{n}\right\}$; thus exactly one coefficient is 1 and the others 0 . It follows that the set of the $n^{k+l}$ tensors $e_{i_{1}} \otimes \ldots \otimes e_{i_{k}} \otimes e^{j_{1}} \otimes \ldots \otimes e^{j_{l}}$ form a basis in $T^{k, l}(V)$, and that the coefficients (1.1) of a tensor $T \in T^{k, l}(V)$ are the coordinates in this basis, i.e.

$$
T=\sum_{i_{1}, \ldots, i_{k}, j_{1}, \ldots, j_{l}} T_{j_{1}, \ldots, j_{l}}^{i_{1}, \ldots, i_{k}} e_{i_{1}} \otimes \ldots \otimes e_{i_{k}} \otimes e^{j_{1}} \otimes \ldots \otimes e^{j_{l}} .
$$

1.5. Change of basis. Consider again a change of basis $f_{i}=\sum_{j} a_{i j} e_{j}$, and thus for the dual bases $f^{i}=\sum_{j} b_{i j} e^{j}$, where as shown above the matrices $A=\left(a_{i j}\right)_{i j}$ and $B=\left(b_{i j}\right)_{i j}$ are related by $B=\left(A^{t}\right)^{-1}=\left(A^{-1}\right)^{t}$. It follows immediately from (1.1) that the coefficients of a tensor $T$ in the new basis are given by

$$
\begin{aligned}
T\left(f^{i_{1}}, \ldots, f^{i_{k}}, f_{j_{1}}, \ldots, f_{j_{l}}\right) & =T\left(\sum_{p_{1}} b_{i_{1} p_{1}} e^{p_{1}}, \ldots, \sum_{q_{l}} a_{j_{l} q_{l}} e_{q_{l}}\right) \\
& =\sum_{p_{1}, \ldots, p_{k}, q_{1}, \ldots, q_{l}} b_{i_{1} p_{1}} \cdots b_{i_{k} p_{k}} a_{j_{1} q_{1}} \cdots a_{j_{l} q_{l}} T_{q_{1}, \ldots, q_{l}}^{p_{1}, \ldots, p_{k}} .
\end{aligned}
$$

Thus, all covariant indices are transformed using the matrix $A=\left(a_{i j}\right)_{i j}$, and the contravariant indices using $B=\left(b_{i j}\right)_{i j}$.

Remark. This is the historical origin of the names covariant and contravariant. The names have stuck, although in the modern point of view, with emphasis on vectors and vector spaces rather than coefficients, they are really not appropriate.

Remark. It may be better to write $a_{i}{ }^{j}$ and $b^{i}{ }_{j}$, to adhere to the convention that a summation index usually appears once as a subscript and once as a superscript, and that other indices should appear in the same place on both sides of an equation. In fact, many authors use the Einstein summation convention, where summation signs generally are omitted, and a summation is implied for each index repeated this way.

Example 1.5.1. Both linear operators $V \rightarrow V$ and bilinear forms on $V$ may be represented by matrices, but, as is well-known from elementary linear algebra, the matrices transform differently under changes of basis. We see here the reason: a linear operator is a tensor of degree $(1,1)$, but a bilinear form is a tensor of degree $(0,2)$.

1.6. Contraction. A tensor $T$ of degree $(1,1)$ may be regarded as a linear operator in $V$ (Example 1.2.4). The trace of this linear operator is called the contraction of $T$.

More generally, let $T$ be a tensor of degree $(k, l)$, with $k, l \geq 1$, and select one contravariant and one covariant argument. By keeping all other arguments fixed, $T$ becomes a bilinear map $V^{*} \times V \rightarrow \mathbb{R}$ of the two selected arguments, i.e. a tensor of degree $(1,1)$ which has a contraction (i.e. trace). This contraction is a real number depending on the remaining $(k-1)+(l-1)$ arguments, and 
it is clearly multilinear in them, so it defines a tensor of degree $(k-1, l-1)$, again called the contraction of $T$.

Given a basis $\left\{e_{1}, \ldots, e_{n}\right\}$ and its dual basis $\left\{e^{1}, \ldots, e^{n}\right\}$, the contraction of a tensor $T$ of degree $(1,1)$ is given by, letting $S$ denote the corresponding linear operator as in (1.2),

$$
\sum_{i}\left\langle e^{i}, S\left(e_{i}\right)\right\rangle=\sum_{i} T\left(e^{i}, e_{i}\right)=\sum_{i} T_{i}^{i} .
$$

More generally, the coefficients of a contraction of a tensor $T$ of degree $(k, l)$ are obtained by summing the $n$ coefficients of $T$ where the indices corresponding to the two selected arguments are equal. For example, contracting the second contravariant and first covariant indices (or arguments) in a tensor $T$ of degree $(2,3)$, we obtain a tensor with coefficients $\widetilde{T}_{j k}^{i}=\sum_{m} T_{m j k}^{i m}$.

Note that we have to specify the indices (or arguments) that we contract. A tensor of degree $(k, l)$ has $k l$ contractions, all of the same degree $(k-1, l-1)$, but in general different.

1.7. Symmetric and antisymmetric tensors. A covariant tensor $T$ of degree 2 is symmetric if $T(v, w)=T(w, v)$ for all $v, w \in V$, and antisymmetric if $T(v, w)=-T(w, v)$ for all $v, w \in V$. (The terms alternating and skew are also used for the latter.) More generally, a covariant tensor $T$ of degree $k$ is symmetric if $T\left(v_{\sigma(1)}, \ldots, v_{\sigma(k)}\right)=T\left(v_{1}, \ldots, v_{k}\right)$ and antisymmetric if $T\left(v_{\sigma(1)}, \ldots, v_{\sigma(k)}\right)=\operatorname{sgn}(\sigma) T\left(v_{1}, \ldots, v_{k}\right)$ for all $v_{1}, \ldots, v_{k} \in V$ and all permutations $\sigma \in \mathfrak{S}_{k}$, the set of all $k$ ! permutations of $\{1, \ldots, k\}$, where $\operatorname{sgn}(\sigma)$ is +1 is $\sigma$ is an even permutation and -1 is $\sigma$ is odd. Equivalently, $T$ is symmetric (antisymmetric) if $T\left(v_{1}, \ldots, v_{k}\right)$ is unchanged (changes its sign) whenever two of the arguments are interchanged. In particular, if $T$ is antisymmetric, then $T\left(v_{1}, \ldots, v_{k}\right)=0$ when two of $v_{1}, \ldots, v_{k}$ are equal. (In fact, this property is equivalent to $T$ being antisymmetric.)

Given a basis of $V$, a tensor $T$ is symmetric (antisymmetric) if and only if its coefficients $T_{i_{1}, \ldots, i_{k}}$ are symmetric (antisymmetric) in the indices. Consequently, a symmetric covariant tensor of degree $k$ is determined by the coefficients $T_{i_{1}, \ldots, i_{k}}$ with $1 \leq i_{1} \leq \cdots \leq i_{k} \leq n$, and an antisymmetric covariant tensor of degree $k$ is determined by the coefficients $T_{i_{1}, \ldots, i_{k}}$ with $1 \leq i_{1}<\cdots<i_{k} \leq n$ (since coefficients with two equal indices automatically vanish). These coefficients may be chosen arbitrarily, and it follows that the linear space of all symmetric covariant tensors of degree $k$ has dimension $\left(\begin{array}{c}n+k-1 \\ k\end{array}\right)$, while the linear space of all antisymmetric covariant tensors of degree $k$ has dimension $\left(\begin{array}{l}n \\ k\end{array}\right)$.

Symmetric and antisymmetric contravariant tensors are defined in the same way. More generally, we may say that a (possibly mixed) tensor is symmetric (or antisymmetric) in two (or several) specified covariant indices, or in two (or several) specified contravariant indices. (It does not make sense to mix covariant and contravariant indices here.)

The tensor product of two symmetric or antisymmetric tensors is, in general, neither symmetric nor antisymmetric. It is, however, possible to symmetrize 
(antisymmetrize) it, and thus define a symmetric tensor product and an antisymmetric tensor product.

The antisymmetric case, which is the most important in differential geometry, is studied in some detail in the next subsection.

1.8. Exterior product. Let $A_{k}(V)$ be the linear space of all antisymmetric covariant tensors of degree $k$. By the last subsection, $\operatorname{dim} A_{k}(V)=\left(\begin{array}{l}n \\ k\end{array}\right)$; in particular, the case $k>n$ is trivial with $\operatorname{dim} A_{k}(V)=0$ and thus $A_{k}(V)=\{0\}$. Note the special cases $A_{0}(V)=\mathbb{R}$ and $A_{1}(V)=V^{*}$. Note also that $A_{n}(V)$ is one-dimensional.

Form further the direct sum $A(V)=\bigoplus_{k=0}^{\infty} A_{k}(V)=\bigoplus_{k=0}^{n} A_{k}(V)$ with dimension $\sum_{0}^{n}\left(\begin{array}{l}n \\ k\end{array}\right)=2^{n}$.

If $T \in A_{k}(V)$ and $U \in A_{l}(V)$, with $k, l \geq 0$, we define their exterior product to be the tensor $T \wedge U \in A_{k+l}(V)$ given by

$T \wedge U\left(v_{1}, \ldots, v_{k+l}\right)=\frac{1}{k ! l !} \sum_{\sigma \in \mathfrak{S}_{k+l}} \operatorname{sgn}(\sigma) T\left(v_{\sigma(1)}, \ldots, v_{\sigma(k)}\right) U\left(v_{\sigma(k+1)}, \ldots, v_{\sigma(k+l)}\right)$.

Remark. Some authors prefer the normalization factor $\frac{1}{(k+l) !}$ instead of $\frac{1}{k ! l !}$, which leads to different constants in some formulas.

It is easily seen that when $k$ or $l$ equals 0 , the exterior product coincides with the usual product of a tensor and a real number.

The exterior product is associative, $T_{1} \wedge\left(T_{2} \wedge T_{3}\right)=\left(T_{1} \wedge T_{2}\right) \wedge T_{3}$, and bilinear (i.e. it satisfies the distributive rules), and it may be extended to a product on $A(V)$ by bilinearity, which makes $A(V)$ into an associative (but not commutative) algebra.

The exterior product is anticommutative, in the sense that

$$
U \wedge T=(-1)^{k l} T \wedge U, \quad T \in A_{k}(V), U \in A_{l}(V) .
$$

If $v_{1}^{*}, \ldots, v_{k}^{*} \in V^{*}=A_{1}(V)$, then $v_{1}^{*} \wedge \cdots \wedge v_{k}^{*} \in A_{k}(V)$, and it follows from (1.6) and induction that

$$
v_{1}^{*} \wedge \cdots \wedge v_{k}^{*}\left(v_{1}, \ldots, v_{k}\right)=\operatorname{det}\left(\left\langle v_{i}^{*}, v_{j}\right\rangle\right)_{i, j=1}^{k} .
$$

If $\left\{e_{1}, \ldots, e_{n}\right\}$ is a basis in $V$, and $\left\{e^{1}, \ldots, e^{n}\right\}$ is the dual basis, then, for any $k \geq 0,\left\{e^{i_{1}} \wedge \cdots \wedge e^{i_{k}}: 1 \leq i_{1}<\ldots i_{k} \leq n\right\}$ is a basis in $A_{k}(V)$ (for $k=0$ we interpret the empty product as 1$)$, and any $T \in A_{k}(V)$ has the expansion

$$
T=\sum_{1 \leq i_{1}<\ldots i_{k} \leq n} T_{i_{1}, \ldots, i_{k}} e^{i_{1}} \wedge \cdots \wedge e^{i_{k}}
$$

as is easily verified using (1.8). The set of all $2^{n}$ products $e^{i_{1}} \wedge \cdots \wedge e^{i_{k}}$, where $k$ is allowed to range form 0 to $n$, is thus a basis in $A(V)$.

Example 1.8.1. In particular, the one-dimensional space $A_{n}(V)$ (where, as usually, $n=\operatorname{dim} V)$ is spanned by $e_{1} \wedge \cdots \wedge e_{n}$, for any basis $\left\{e_{1}, \ldots, e_{n}\right\}$ in $V$. 
If $\left\{f_{1}, \ldots, f_{n}\right\}$ is another basis in $V$, with $f_{i}=\sum_{j} a_{i j} e_{j}$, then the basis change in $A_{n}(V)$ is given by

$$
\begin{aligned}
f_{1} \wedge \cdots \wedge f_{n} & =\sum_{j_{1}, \ldots, j_{n}}\left(\prod_{i=1}^{n} a_{i j_{i}}\right) e_{j_{1}} \wedge \cdots \wedge e_{j_{n}} \\
& =\sum_{\sigma \in \mathfrak{S}_{n}}\left(\prod_{i=1}^{n} a_{i \sigma(i)}\right) e_{\sigma(1)} \wedge \cdots \wedge e_{\sigma(n)} \\
& =\sum_{\sigma \in \mathfrak{S}_{n}}\left(\prod_{i=1}^{n} a_{i \sigma(i)}\right) \operatorname{sgn}(\sigma) e_{1} \wedge \cdots \wedge e_{n} \\
& =\operatorname{det}(A) e_{1} \wedge \cdots \wedge e_{n},
\end{aligned}
$$

using the matrix $A=\left(a_{i j}\right)_{i j}$.

1.9. Tensors over a Euclidean space. An inner product on $V$ is a bilinear form $V \times V \rightarrow \mathbb{R}$ that is positive definite (and thus symmetric), in other words a special tensor of degree $(0,2)$.

Now suppose that $V$ is a Euclidean space, i.e. a vector space equipped with a specific inner product, which we denote both by $\langle$,$\rangle and g$. Then there is a natural isomorphism between $V$ and $V^{*}$, where $v \in V$ can be identified with the linear functional $h(v): w \mapsto\langle v, w\rangle$. Using this identification we define a dual inner product $g^{*}$ in $V^{*}$ by $g^{*}\left(v^{*}, w^{*}\right)=g(v, w)$ when $v^{*}=h(v), w^{*}=h(w)$, thus making $h$ an isometry. Note that $g^{*}$ is a bilinear form on $V^{*}$ and thus a contravariant tensor of degree $(2,0)$. We use the alternative notation $\langle$,$\rangle for$ $g^{*}$ too; thus $\langle$,$\rangle is really used in three senses, viz. for the inner products in$ $V$ and $V^{*}$, and for the pairing between $V$ and $V^{*}$. There is no great danger of confusion, however, because the three interpretations coincide if we use the identification between $V$ and $V^{*}:\langle v, w\rangle=\langle h(v), w\rangle=\langle h(v), h(w)\rangle$.

Given a basis $\left\{e_{1}, \ldots, e_{n}\right\}$, we denote the coefficients of the inner products $g$ and $g^{*}$ by $g_{i j}$ and $g^{i j}$, respectively. If $v \in V$ corresponds to $v^{*}=h(v) \in V^{*}$, then the coefficients of $v$ and $v^{*}$ are related by

$$
v_{i}^{*}=\left\langle v^{*}, e_{i}\right\rangle=\left\langle v, e_{i}\right\rangle=\left\langle\sum_{j} v^{j} e_{j}, e_{i}\right\rangle=\sum_{j} v^{j} g\left(e_{j}, e_{i}\right)=\sum_{j} g_{i j} v^{j} .
$$

We thus obtain the coefficients of $v^{*}$ by multiplying the coefficients of $v$ by the matrix $\left(g_{i j}\right)_{i j}$. Similarly, the coefficients of $v$ are obtained by multiplying the coefficients of $v^{*}$ by the matrix $\left(g^{i j}\right)_{i j}$ :

$$
v^{i}=\left\langle e^{i}, v\right\rangle=\left\langle e^{i}, v^{*}\right\rangle=\left\langle e^{i}, \sum_{j} v_{j}^{*} e^{j}\right\rangle=\sum_{j} g^{i j} v_{j}^{*} .
$$

Since the operations in (1.10) and (1.11) are inverse to to each other, it follows that the matrix $\left(g^{i j}\right)_{i j}$ is the matrix inverse of $\left(g_{i j}\right)_{i j}$.

The operations of going between $v \in V$ and the corresponding $v^{*}=h(v) \in$ $V^{*}$ are known as lowering and raising the index. Note that, by (1.10), lowering the index may be regarded as taking the tensor product with $g$ followed by a 
contraction. Similarly, raising an index may be regarded as taking the tensor product with $g^{*}$ followed by a contraction.

The isomorphism between $V$ and $V^{*}$, or equivalently between tensors of degree $(1,0)$ and $(0,1)$, extends to tensors of higher degree. Indeed, a tensor of degree $(k, l)$ is defined as a multilinear form on $V^{* k} \times V^{l}$, but we may now identify such a form with a multilinear form on $V^{k+l}$ (or on any other product of $k+l$ spaces $V$ or $V^{*}$ ), and thus there is a natural correspondence $T^{k, l}(V) \cong T^{0, k+l}(V)$ between tensors of degree $(k, l)$ and covariant tensors of degree $k+l$. Thus it suffices to consider covariant tensors only (or, which in some cases is better, contravariant tensors only). The relations (1.10) and (1.11) extend to tensors of higher degree; again they may be regarded as taking tensor products with $g$ or $g^{*}$ followed by a contraction; now several indices may be lowered or raised simultaneously, independently of each other, by taking the tensor product with several copies of $g$ and $g^{*}$ and making the appropriate contractions. Note, however, that we still have to keep track of the order of the indices.

Example 1.9.1. A covariant tensor $T$ of degree $(0,2)$ corresponds to two tensors of degree $(1,1)$, obtained by raising different indices, and a contravariant tensor of degree $(2,0)$ obtained by raising both indices (in any order). If $T$ has coefficients $t_{i j}$, these tensors have coefficients $T_{j}^{i}=\sum_{k} g^{i k} T_{k j}, T_{i}{ }^{j}=\sum_{k} g^{j k} T_{i k}$ and $T^{i j}=\sum_{k, l} g^{i k} g^{j l} T_{k l}$, respectively.

Example 1.9.2. Recall from Example 1.2.4 that the identity mapping $V \rightarrow V$ can be regarded as a tensor $\iota \in T^{1,1}(V)$ with coefficients $\delta_{j}^{i}$. Lowering or raising an index we obtain tensors with the coefficients $g_{i j}$ and $g^{i j}$, respectively, i.e. $g$ and $g^{*}$. In other words, $g, \iota$ and $g^{*}$ correspond to each other under raising and lowering of indices. (In this case, the two tensors of degree $(1,1)$ obtained by raising different indices in $g$ coincide by symmetry.)

Example 1.9.3. If $\left\{e_{1}, \ldots, e_{n}\right\}$ is an orthonormal basis in $V$, i.e. $g_{i j}=\delta_{i j}$, we have $g^{i j}=\delta_{i j}$ (better written $\delta^{i j}$ ) too. Thus the dual basis $\left\{e^{1}, \ldots, e^{n}\right\}$ is also orthonormal. Moreover, the basis elements correspond to each other under the identification of $V$ and $V^{*}: h\left(e_{i}\right)=e^{i}$. Raising and lowering of indices becomes trivial; the coefficients remain the same regardless of the position of the indices. For example, for a tensor of degree 2 as in Example 1.9.1, $T_{i j}=T^{i}{ }_{j}=T_{i}^{j}=T^{i j}$.

\section{TENSORS ON A MANIFOLD}

In this section, $M$ is a differentiable manifold of dimension $n$. The linear space of all infinitely differentiable real-valued functions on $M$ is denoted by $C^{\infty}(M)$ (another common notation is $\mathcal{D}(M)$ ), and the space of all vector fields is denoted by $\mathcal{X}(M)$.

$T_{p}(M)$ is the tangent space at $p \in M$, and $T(M)$ is the tangent bundle, i.e. the (disjoint) union of all $T_{p}(M)$. 
2.1. Cotangent vectors. The dual space $T_{p}^{*}(M)$ of $T_{p}(M)$ is called the cotangent space at $p$, and its elements, i.e. the linear functionals on $T_{p}(M)$, are called cotangent vectors.

If $f$ is a smooth function on $M$, or at least in a neighbourhood of $p$, the mapping $X \mapsto X(f)$ is defined for all $X \in T_{p}(M)$; this mapping is clearly linear and thus defines a cotangent vector denoted by $d f_{p}$ or just $d f$. In other words, $d f_{p} \in T_{p}^{*}(M)$ is defined by

$$
\left\langle d f_{p}, X\right\rangle=X(f), \quad X \in T_{p}(M) .
$$

Remark. The general definition of the differential $d f$ of a differentiable mapping between two manifolds defines $d f_{p}$ as a linear mapping between the respective tangent spaces at $p$ and $f(p)$. With the standard identification of the tangent space $T_{f(p)}(\mathbb{R})$ with $\mathbb{R}$, the two definitions of $d f_{p}$ coincide (which justifies the use of the same notation for both).

If $\left(x^{1}, \ldots, x^{n}\right)$ is a coordinate system in a neighbourhood of $p$, the partial derivatives $\partial_{i}=\partial / \partial x^{i}, i=1, \ldots, n$, form a basis in $T_{p}(M)$. Moreover, the differentials $d x^{i}$ of the coordinate functions satisfy by (2.1)

$$
\left\langle d x^{i}, \partial / \partial x^{j}\right\rangle=\frac{\partial x^{i}}{\partial x^{j}}=\delta_{i j} .
$$

Consequently, the dual basis of $\left\{\partial_{1}, \ldots, \partial_{n}\right\}$ is $\left\{d x^{1}, \ldots, d x^{n}\right\}$. Note that in this basis, the differential $d f$ of a function $f$ has the coefficients $\left\langle d f, \partial / \partial x^{i}\right\rangle=$ $\partial f / \partial x^{i}$, i.e.

$$
d f=\sum_{i} \frac{\partial f}{\partial x^{i}} d x^{i} .
$$

2.2. Frames. A frame $\left\{E_{1}, \ldots, E_{n}\right\}$ in an open subset $U$ of $M$ is a collection of $n$ vector fields on $U$ that are linearly independent at each $q \in U$, and thus form a basis of each tangent space $T_{q}(M)$. At each $q \in U$ there is a dual basis $\left\{E_{q}^{1}, \ldots, E_{q}^{n}\right\}$ in $T_{q}^{*}(M)$, and the collection of the $n$ functions $E^{i}: q \mapsto E_{q}^{i}$ is called the dual frame.

If $\left(x^{1}, \ldots, x^{n}\right)$ is a coordinate system in an open set $U$, the partial derivatives $\partial / \partial x^{1}, \ldots, \partial / \partial x^{n}$ form a frame in $U$, and the dual frame is, as shown above, given by the differentials $d x^{1}, \ldots, d x^{n}$. (This is called a coordinate frame and is perhaps the most important example of a frame, but other frames are sometimes convenient; for example it is in a Riemannian manifold often useful to consider orthonormal frames.)

Remark. It is not always possible to define a frame on all of $M$, so we have to consider subsets $U$. (Manifolds that have a globally defined frame are known as parallelizable.) For example, on the sphere $S^{2}$ (or any even-dimensional sphere), there does not even exist a vector field that is everywhere nonzero.

2.3. Tensors at a point. At each point $p \in M$, and for each $k, l \geq 0$, we define the tensor space $T_{p}^{k, l}(M)$ to be the space $T^{k, l}\left(T_{p}(M)\right)$ of tensors over the tangent space. In particular, a tensor at $p$ of degree $(0,0)$ is a real number, a tensor at $p$ of degree $(1,0)$ is a tangent vector, and a tensor at $p$ of degree $(1,0)$ is a cotangent vector. 
2.4. Tensor fields. Let $k$ and $l$ be non-negative integers. A rough tensor field of degree $(k, l)$ on $M$ is a function $p \mapsto T(p)$ that for every $p \in M$ assigns a tensor $T(p) \in T_{p}^{k, l}(M)$. We similarly define rough tensor fields on a subset of $M$.

In particular, a rough tensor field of degree $(0,0)$ is any real-valued function, and a rough tensor field of degree $(1,0)$ is a rough vector field, i.e. a function that to every point assigns a tangent vector at that point, without any assumption on smoothness (or even continuity). Just as for vector fields, we want to add a smoothness assumption for tensor fields; this can be done as follows.

Let $\left\{E_{1}, \ldots, E_{n}\right\}$ be a frame in a neighbourhood $U$ of $p \in M$, with the dual frame $\left\{E^{1}, \ldots, E^{n}\right\}$. A rough tensor field $T$ of degree $(k, l)$ on $U$ can be described by its coefficients

$$
T_{j_{1}, \ldots, j_{l}}^{i_{1}, \ldots, i_{k}}(q)=T\left(E^{i_{1}}, \ldots, E^{i_{k}}, E_{j_{1}}, \ldots, E_{j_{l}}\right)(q)
$$

(evaluated pointwise); the coefficients are thus real-valued functions on $U$. We say that $T$ is smooth at $p$ if all coefficients are smooth functions in a neighbourhood of $p$. Note that if $\left\{F_{1}, \ldots, F_{n}\right\}$ is another frame on a (possibly different) neighbourhood of $p$, the corresponding change of basis in each $T_{q}(M)$ is described by a matrix-valued function $A=\left(a_{i j}\right)_{i j}$ (defined on the intersection of the two neighbourhoods); clearly each $a_{i j}$ a smooth fuction, and thus also $B=\left(A^{-1}\right)^{t}$ has smooth entries. It follows from (1.5) that if $T$ has smooth coefficients at $p$ for $\left\{E_{1}, \ldots, E_{n}\right\}$, it has so for $\left\{F_{1}, \ldots, F_{n}\right\}$ too, and conversely; consequently the definition of smooth does not depend on the chosen frame.

A smooth tensor field on $M$ (or on an open subset) is a rough tensor field that is smooth at every point; in other words, the coefficients for each frame are smooth (on the subset where the frame is defined). Note that it suffices to check this for any collection of frames whose domains cover $M$. (For example, one can use a collection of coordinate frames whose domains cover $M$.)

A smooth tensor field is usually called just a tensor field or even a tensor.

Example 2.4.1. A tensor field of degree $(0,0)$, also called a scalar tensor field, is the same as a smooth real-valued function.

Example 2.4.2. A tensor field of degree $(1,0)$ is the same as a vector field.

Example 2.4.3. A tensor field of degree $(0,1)$ is a smooth assignment of a cotangent vector at each point of $M$. This is usually called a 1-form, for reasons explained in Section 3.

Example 2.4.4. If $\left\{E_{1}, \ldots, E_{n}\right\}$ is a frame on an open set $U$, the elements $E^{1}, \ldots, E^{n}$ of the dual frame are $n$ tensor fields of degree $(0,1)$ on $U$, which trivially are smooth; thus they are 1-forms on $U$.

In particular, for any coordinate system $\left(x^{1}, \ldots, x^{n}\right)$ in an open set $U$, the differentials $d x^{1}, \ldots, d x^{n}$ are 1-forms on $U$.

Example 2.4.5. More generally, the differential $d f$ of any smooth function $f$ is a 1 -form. 
Example 2.4.6. A Riemannian metric in $M$ is by definition a tensor field of degree $(0,2)$ which at each point is positive definite (as a bilinear form on $\left.T_{p}(M)\right)$.

2.5. Change of basis. As said above, the coefficients of a tensor with respect to two different frames (on the same set) are related by (1.5), where $\left(a_{i j}\right)$ and $\left(b_{i j}\right)$ are matrices (of functions) describing the change of frame. In the special case of two coordinate frames given by coordinate systems $\left(x^{1}, \ldots, x^{n}\right)$ and $\left(y^{1}, \ldots, y^{n}\right)$, we have $a_{i j}=\partial x^{j} / \partial y^{i}$ and $b_{i j}=\partial y^{i} / \partial x^{j}$, and thus, if the tensor has coefficients $T_{j_{1}, \ldots, j_{l}}^{i_{1}, \ldots, i_{k}}$ for $\left(x^{1}, \ldots, x^{n}\right)$, its coefficients for $\left(y^{1}, \ldots, y^{n}\right)$ are given by

$$
\sum_{p_{1}, \ldots, p_{k}, q_{1}, \ldots, q_{l}} \frac{\partial y^{i_{1}}}{\partial x^{p_{1}}} \cdots \frac{\partial y^{i_{k}}}{\partial x^{p_{k}}} \frac{\partial y^{q_{1}}}{\partial x^{j_{1}}} \cdots \frac{\partial y^{q_{l}}}{\partial x^{j_{l}}} T_{q_{1}, \ldots, q_{l}}^{p_{1}, \ldots, p_{k}} .
$$

2.6. Algebraic operations on tensor fields. The sum of two tensor fields of the same degree, the tensor product of two arbitrary tensor fields, and contractions of a tensor fields are defined by applying the definitions of Section 1 at each point $p$. Clearly, the results are (smooth) tensor fields of the appropriate degrees.

2.7. Tensor fields as $C^{\infty}$-linear maps. A rough tensor field $\omega$ of degree $(0,1)$ defines a mapping from $\mathcal{X}(M)$ to the space of all real-valued functions on $M$ by $X \mapsto \omega(X)$ (evaluated pointwise), and $\omega$ is smooth if and only if $\omega(X)$ is a smooth function for every vector field $X$, i.e. if this mapping maps $\mathcal{X}(M)$ into $C^{\infty}(M)$.

Moreover, the mapping $X \mapsto \omega(X)$ is $C^{\infty}$-linear, i.e. $\omega(f X)=f \omega(X)$ for every $f \in C^{\infty}(M)$. Conversely, every $C^{\infty}$-linear mapping $\mathcal{X}(M) \rightarrow C^{\infty}(M)$ is given in this way by a (unique) 1 -form.

Proof. If $S: \mathcal{X}(M) \rightarrow C^{\infty}(M)$ is $C^{\infty}$-linear and $p \in M$, we first show that $X(p)=0$ implies $S(X)(p)=0$. Indeed, if $\left\{E_{1}, \ldots, E_{n}\right\}$ is any frame in a neighbourhood $U$ of $p$, then $X=\sum_{i} f_{i} E_{i}$ in $U$, where $f_{i}$ are smooth functions in $U$ with $f_{i}(p)=0$. Choose a function $\varphi \in C^{\infty}(M)$ with support in $U$ such that $\varphi(p)=1$, and let $g_{i}(q)=\varphi(q) f_{i}(q)$ when $q \in U$ and $g_{i}(q)=0$ otherwise; then $g_{i} \in C^{\infty}(M)$. Similarly, define vector fields $Y_{i} \in \mathcal{X}(M)$ such that $Y_{i}=\varphi E_{i}$ on $U$. Then, $\varphi^{2} X=\sum_{i} g_{i} Y_{i}$ on $M$. Thus, at $p$,

$$
S(X)=\varphi^{2} S(X)=S\left(\varphi^{2} X\right)=S\left(\sum_{i} g_{i} Y_{i}\right)=\sum_{i} g_{i} S\left(Y_{i}\right)=0,
$$

since $g_{i}(p)=0$ for all $i$.

Consequently, using linearity, $S(X)(p)$ depends only on $X(p)$, and thus there is a cotangent vector $\omega_{p} \in T_{p}^{*}(M)$ such that $S(X)(p)=\omega_{p}(X(p))$; in other words there is a rough tensor field $\omega$ of degree $(0,1)$ that defines $S$. Since $S$ maps into $C^{\infty}(M), \omega$ is smooth.

The argument extends to tensor fields of higher degrees and multilinear mappings as follows. 
Theorem 2.7.1. Let $T: \mathcal{X}^{*}(M)^{k} \times \mathcal{X}(M)^{l} \rightarrow C^{\infty}(M)$ be a multilinear mapping, where $k, l \geq 0$. Then the following are equivalent:

(i) There exists a tensor field $\widetilde{T}$ of degree $(k, l)$ such that (at every point) $T\left(\omega_{1}, \ldots, \omega_{k}, X_{1}, \ldots, X_{l}\right)=\widetilde{T}\left(\omega_{1}, \ldots, \omega_{k}, X_{1}, \ldots, X_{l}\right)$.

(ii) $T$ is $C^{\infty}$-multilinear.

(iii) If $\omega_{1}, \ldots, \omega_{k}, \omega_{1}^{\prime}, \ldots, \omega_{k}^{\prime}, X_{1}, \ldots, X_{l}, X_{1}^{\prime}, \ldots, X_{l}^{\prime}$ are 1 -forms and vector fields, respectively, such that $\omega_{i}(p)=\omega_{i}^{\prime}(p)$ and $X_{i}(p)=X_{i}^{\prime}(p)$ at some $p \in M$, then $T\left(\omega_{1}, \ldots, \omega_{k}, X_{1}, \ldots, X_{l}\right)(p)=T\left(\omega_{1}^{\prime}, \ldots, \omega_{k}^{\prime}, X_{1}^{\prime}, \ldots, X_{l}^{\prime}\right)(p)$.

(iv) If one of the fields $\omega_{1}, \ldots, \omega_{k}, X_{1}, \ldots, X_{l}$ vanishes at a point $p$, then $T\left(\omega_{1}, \ldots, \omega_{k}, X_{1}, \ldots, X_{l}\right)(p)=0$.

Furthermore, if $T$ is a tensor field of degree $(1, l)$ for some $l \geq 0$, then by Example 1.2.5, it defines at each $p \in M$ a multilinear map $\widetilde{T}: T_{p}^{*}(M)^{l} \rightarrow$ $T_{p}(M)$ such that for any vector fields $X_{1}, \ldots, X_{l}$ and a 1 -form $\omega$, we have at every $p$

$$
T\left(\omega, X_{1}, \ldots, X_{l}\right)=\left\langle\omega, \widetilde{T}\left(X_{1}, \ldots, X_{l}\right)\right\rangle .
$$

It follows that, as $p$ varies, $\widetilde{T}\left(X_{1}, \ldots, X_{l}\right)$ defines a vector field on $M$; evidently $\widetilde{T}$ is a $C^{\infty}$-multilinear mapping $\mathcal{X}(M)^{l} \rightarrow \mathcal{X}(M)$. Conversely every such mapping defines by (2.4) a $C^{\infty}$-multilinear mapping $\mathcal{X}^{*}(M) \times \mathcal{X}(M)^{l} \rightarrow C^{\infty}(M)$, and thus by Theorem 2.7.1 a tensor field of degree $(1, l)$. This yields the following variant of Theorem 2.7.1.

Theorem 2.7.2. Let $T: \mathcal{X}(M)^{l} \rightarrow \mathcal{X}(M)$ be a multilinear mapping, where $l \geq 0$. Then the following are equivalent.

(i) There exists a tensor field $\widetilde{T}$ of degree $(1, l)$ such that $(2.4)$ holds.

(ii) $T$ is $C^{\infty}$-multilinear.

(iii) If $X_{1}, \ldots, X_{l}, X_{1}^{\prime}, \ldots, X_{l}^{\prime}$ are vector fields such that $X_{i}(p)=X_{i}^{\prime}(p)$ at some $p \in M$, then $T\left(X_{1}, \ldots, X_{l}\right)(p)=T\left(X_{1}^{\prime}, \ldots, X_{l}^{\prime}\right)(p)$.

(iv) If one of the fields $X_{1}, \ldots, X_{l}$ vanishes at a point $p$, then $T\left(X_{1}, \ldots, X_{l}\right)(p)$ $=0$.

Example 2.7.1. A Riemannian metric can equivalently be defined as a $C^{\infty}$ bilinear mapping $g: \mathcal{X}(M)^{2} \rightarrow C^{\infty}(M)$ such that $g(X, X)>0$ at every point where $X \neq 0$.

Example 2.7.2. The Lie product $(X, Y) \mapsto[X, Y]$ of vector fields is a bilinear mapping $\mathcal{X}(M)^{2} \rightarrow \mathcal{X}(M)$, but it is not $C^{\infty}$-bilinear, and is thus not a tensor.

Example 2.7.3. An affine connection on $M$ is a bilinear mapping $\mathcal{X}(M)^{2} \rightarrow$ $\mathcal{X}(M)$ which it is not $C^{\infty}$-bilinear, and is thus not a tensor. This is also seen because its coefficients in a coordinate system (or for a general frame) do not obey the transformation rule (1.5).

Example 2.7.4. On the other hand, if $\nabla$ is an affine connection and $Y$ is a fixed vector field, then $X \mapsto \nabla_{X} Y$ is a $C^{\infty}$-linear map $\mathcal{X}(M) \rightarrow \mathcal{X}(M)$, and can thus be regarded as a tensor field of degree $(1,1)$. This tensor field is denoted by $\nabla Y$. 
Example 2.7.5. If $\nabla$ is an affine connection, then a simple calculation shows that $(X, Y) \mapsto \nabla_{X} Y-\nabla_{Y} X-[X, Y]$ defines a $C^{\infty}$-bilinear map $\mathcal{X}(M)^{2} \rightarrow$ $\mathcal{X}(M)$, and thus a tensor field of degree $(1,2)$. This is called the torsion tensor (of the connection). The connection is called symmetic when its torsion vanishes, (The standard connection on a Riemannian manifold is symmetric, and thus there is no interest in the torsion tensor in Riemannian geometry.)

Example 2.7.6. If $\nabla$ is an affine connection, then a somewhat longer calculation shows that

$$
(X, Y, Z) \mapsto R(X, Y) Z:=-\nabla_{X} \nabla_{Y} Z+\nabla_{Y} \nabla_{X} Z+\nabla_{[X, Y]} Z
$$

defines a $C^{\infty}$-trilinear map $\mathcal{X}(M)^{3} \rightarrow \mathcal{X}(M)$, and thus a tensor field of degree $(1,3)$. This is the Riemann curvature tensor, generally denoted by $R$. (Many authors choose the opposite sign.)

We can contract the curvature tensor to a tensor of degree $(0,2)$. This can be done in three different ways, but for a Riemannian connection, one of them yields the zero tensor (by symmetry), and is thus not very interesting. (It is non-zero for other affine connections, in general, but I do not know any use of this contraction.) The other two possibilities differ by sign only, again by symmetry because $R(X, Y) Z=-R(Y, X) Z$ from the definition, and one of them is called the Ricci tensor; we define it by contraction of the contravariant and the second covariant index in $R$. (All authors make the choice depending on their choice of sign for $R$, so that the sign of the Ricci tensor always is the same; for example, the Ricci tensor for a sphere is a positive multiple of the metric tensor. However, do Carmo [2] uses a non-standard normalization factor $1 /(n-1)$ in his definition.)

2.8. Tensor bundles. The tensor bundle $T^{k, l}(M)$ of degree $(k, l)$ is defined as the disjoint union of all tensor spaces $T_{p}^{k, l}(M)$. Each coordinate system $\left(x^{1}, \ldots, x^{n}\right)$ on an open set $U$ defines a coordinate system on the corresponding part $T_{p}^{k, l}(U)=\bigcup_{p \in U} T_{p}^{k, l}$ of $T_{p}^{k, l}(M)$ by taking as coordinates of a tensor $T \in$ $T_{p}^{k, l}, p \in U$, the $n$ coordinates $x^{i}(p)$ followed by the $n^{k+l}$ coefficients of $T$ for the basis $\left\{\partial / \partial x^{1}, \ldots, \partial / \partial x^{n}\right\}$. It is easily seen that these coordinates systems define a differentiable structure on $T^{k, l}(M)$, and thus the tensor bundle $T^{k, l}(M)$ is a differentiable manifold with dimension $n+n^{k+l}$.

Letting $\pi: T^{k, l}(M) \rightarrow M$ denote the natural projection mapping a tensor $T \in T_{p}^{k, l}$ to $p$, we can give an alternative (but equivalent) definition of a tensor field of degree $(k, l)$ as a differentiable function $p \mapsto T(p)$ of $M$ into $T^{k, l}(M)$, such that $\pi(T(p))=p$ for every $p$. (Such functions are known as sections in the bundle.

Example 2.8.1. $T^{0,0}(M)=M \times \mathbb{R}$.

Example 2.8.2. $T^{1,0}(M)$ is the tangent bundle $T(M)$.

Example 2.8.3. $T^{0,1}(M)$ is called the cotangent bundle.

Remark. More generally, a vector bundle over a manifold $M$ is a disjoint union of a collection $\left\{V_{p}\right\}_{p \in M}$ of vector spaces of a fixed dimension $N$ which is 
equipped with a differentiable structure on the bundle that can be defined by a collection of open sets $U$ covering $M$ and for each of them a set of $N$ functions $e_{1}, \ldots e_{N}$ from $U$ into the bundle, such that, at each $p \in U, e_{1}(p), \ldots e_{N}(p)$ form a basis in $V_{p}$; a coordinate system for the bundle is defined by taking as coordinates of $v \in V_{p}$ the coordinates of $p$ in a coordinate system on $M$, followed by the coordinates of $v$ in the basis $e_{1}(p), \ldots e_{N}(p)$. (Hence, a vector bundle is locally isomorphic to a product $U \times \mathbb{R}^{N}, U \subset M$.) The vector space $V_{p}$ is called the fiber of the bundle at $p$. Sections of the bundle are defined as above, thus yielding generalizations of vector fields and tensor fields.

Algebraic operations on vector spaces like taking the dual, direct sum, tensor product, ..., yield (applied to the fibers) corresponding operations on vector bundles, generalizing the constructions above.

For another example of a vector bundle, suppose that $N$ is another manifold and $f: M \rightarrow N$ a differentiable function, and define $V_{p}=T_{f(p)}(N)$; this defines a vector bundle over $M$ (known as the pull-back of the tangent bundle over $N)$. If further $f$ is an immersion, we can identify $T_{p}(M)$ with the subspace $d f_{p}\left(T_{p}(M)\right.$ of $T_{f(p)}(N)$, and thus regard $T(M)$ as a subbundle of the pull-back; if moreover $N$ is a Riemannian manifold, we can define another bundle over $M$, the normal bundle of the immersion, as the subbundle of the pull-back whose fiber $V_{p}$ at $p$ is the orthogonal complement of $d f_{p}\left(T_{p}(M)\right.$ in $T_{f(p)}(N)$, see [2, Section 6.3].

2.9. Covariant derivation of tensor fields. In any differentiable manifold, we can differentiate functions by a vector field (or by a tangent vector at a single point). Given an affine connection, we can also differentiate vector fields (the covariant derivative). This extends to tensor fields of arbitrary degrees as follows.

Let $\mathcal{T}^{k, l}(M)$ denote the linear space of all tensor fields of degree $(k, l)$ om M. (Thus $\mathcal{T}^{0,0}(M)=C^{\infty}(M)$ and $\mathcal{T}^{1,0}(M)=\mathcal{X}(M)$.)

Theorem 2.9.1. If $M$ is a differentiable manifold with an affine connection $\nabla$, there is a unique extension of $\nabla$ to an operator $(X, T) \mapsto \nabla_{X} T$ defined for any vector field $X$ and tensor field $T$ (of arbitrary degree) such that:

(i) For each $k, l \geq 0, \nabla$ is a bilinear mapping $\mathcal{X}(M) \times \mathcal{T}^{k, l}(M) \rightarrow$ $\mathcal{T}^{k, l}(M)$. (Thus $\nabla_{X} T$ has the same degree as $T$.)

(ii) $\nabla_{X} T$ is further $C^{\infty}$-linear in $X: \nabla_{f X} T=f \nabla_{X} T$ for any $f \in C^{\infty}(M)$ and tensor field $T$.

(iii) $\nabla_{X}(f T)=f \nabla_{X} T+(X f) T$ for any $f \in C^{\infty}(M)$ and tensor field $T$.

(iv) $\nabla_{X} f=X f$ when $f \in C^{\infty}(M)$, i.e. when $f$ is a tensor field of degree $(0,0)$.

(v) $\nabla_{X} Y$ is the vector field given by the connection when $Y$ is a vector field, i.e. when $Y$ is a tensor field of degree $(1,0)$.

(vi) $\nabla_{X}(T \otimes U)=\left(\nabla_{X} T\right) \otimes U+T \otimes \nabla_{X} U$ for any two tensor fields $T$ and $U$.

(vii) $\nabla_{X}$ commutes with contractions. 
This operator $\nabla$ is explicitly given for a 1-form $\omega$ by

$$
\left\langle\nabla_{X} \omega, Y\right\rangle=X(\langle\omega, Y\rangle)-\left\langle\omega, \nabla_{X} Y\right\rangle, \quad X, Y \in \mathcal{X}(M),
$$

and, in general, for a tensor field of degree $(k, l)$ by

$$
\begin{aligned}
& \nabla_{X} T\left(\omega_{1}, \ldots, \omega_{k}, Y_{1}, \ldots, Y_{l}\right)=\nabla_{X}\left(T\left(\omega_{1}, \ldots, \omega_{k}, Y_{1}, \ldots, Y_{l}\right)\right) \\
& \quad-T\left(\nabla_{X} \omega_{1}, \ldots, \omega_{k}, Y_{1}, \ldots, Y_{l}\right)-\cdots-T\left(\omega_{1}, \ldots, \nabla_{X} \omega_{k}, Y_{1}, \ldots, Y_{l}\right) \\
& \quad-T\left(\omega_{1}, \ldots, \omega_{k}, \nabla_{X} Y_{1}, \ldots, Y_{l}\right)-\cdots-T\left(\omega_{1}, \ldots, \omega_{k}, Y_{1}, \ldots, \nabla_{X} Y_{l}\right)
\end{aligned}
$$

for any 1 -forms $\omega_{1}, \ldots, \omega_{k}$ and vector fields $Y_{1}, \ldots, Y_{l}$.

Finally, the value of $\nabla_{X} T$ at a point $p$ depends only on $X_{p}$ and the values of $T$ in a neigbourhood of $p$.

Proof. Suppose that $\nabla$ is such an extension. If $\omega$ is a 1-form and $Y$ a vector field, then $\omega \otimes Y$ is a tensor field of degree $(1,1)$, whose contraction is the function $\langle\omega, Y\rangle=\omega(Y)$. By (vi), $\nabla_{X}(\omega \otimes Y)=\left(\nabla_{Y} \omega\right) \otimes Y+\omega \otimes\left(\nabla_{X} Y\right)$, and thus by contracting, using (vii),

$$
\nabla_{X}\langle\omega, Y\rangle=\left\langle\nabla_{X} \omega, Y\right\rangle+\left\langle\omega, \nabla_{X} Y\right\rangle .
$$

By (iv), this yields (2.5).

Similarly, if $T$ is of degree $(k, l), \omega_{1}, \ldots, \omega_{k}$ are 1 -forms and $X_{1}, \ldots, X_{l}$ are vector fields, then the function $T\left(\omega_{1}, \ldots, \omega_{k}, X_{1}, \ldots, X_{l}\right)$ may be obtained by contracting all indices (in the correct combination) of the tensor product $T \otimes$ $\omega_{1} \otimes \cdots \otimes \omega_{k} \otimes X_{1} \otimes \cdots \otimes X_{l}$. Using again (vi) and (vii), we obtain (2.6).

This shows uniqueness of $\nabla_{X}$. Conversely, we may define $\nabla_{X} T$ for all tensor fields $T$ by (2.6), where $\nabla_{X} \omega$ are defined by (2.5). A routine verification shows that $\nabla_{X}$ satisfies all properties listed in the theorem. (Note that, using (iv), (iii) is a special case of (vi).)

The operator $\nabla$ is called covariant derivation. Let $T$ be a tensor field of degree $(k, l)$. Since $X \mapsto \nabla_{X} T$ is $C^{\infty}$-linear in $X$, we can also (in the usual way) define $\nabla_{v} T$ for a single tangent vector $v \in T(M)$. Moreover, the mapping $\left(\omega_{1}, \ldots, \omega_{k}, X_{1}, \ldots, X_{l}, X\right) \mapsto \nabla_{X} T\left(\omega_{1}, \ldots, \omega_{k}, X_{1}, \ldots, X_{l}\right)$ is $C^{\infty}$-multilinear, and defines thus by Theorem 2.7.1 a tensor of degree $(k, l+1)$, called the covariant differential of $T$. (Cf. Example 2.7.4.)

Let $\left(x^{1}, \ldots, x^{n}\right)$ be a coordinate system (in some open set). Let $\partial_{i}=\partial / \partial x^{i}$ and denote the coefficients of the connection by

$$
\Gamma_{i j}^{k}=\left\langle d x^{k}, \nabla_{\partial_{j}} \partial_{i}\right\rangle .
$$

It is traditional to denote partial differentiation (with respect to a particular coordinate system) by a comma, thus $f_{, i}=\partial_{i} f=\partial f / \partial x^{i}$ for a function $f$, and to denote covariant differentiation by a semicolon, thus the components of $\nabla T$, where $T \in \mathcal{T}^{k, l}(M)$, are written $T_{j_{1}, \ldots, j_{l} ; j}^{i_{1}, \ldots, i_{k}}$. For a vector field $X=\sum_{i} X^{i} \partial_{i}$ we have

$\nabla_{\partial_{j}} X=\nabla_{\partial_{j}}\left(\sum_{i} X^{i} \partial_{i}\right)=\sum_{i}\left(\left(\partial_{j} X^{i}\right) \partial_{i}+X^{i} \nabla_{\partial_{j}} \partial_{i}\right)=\sum_{i} X_{, j}^{i} \partial_{i}+\sum_{i k} X^{i} \Gamma_{i j}^{k} \partial_{j}$ 
and thus

$$
X_{; j}^{i}=X_{, j}^{i}+\sum_{m} X^{m} \Gamma_{m j}^{i}
$$

It now follows from (2.5) that for a 1 -form $\omega$,

$$
\omega_{i ; j}=\left\langle\nabla_{\partial_{j}} \omega, \partial_{i}\right\rangle=\omega_{i, j}-\sum_{k} \omega_{k} \Gamma_{i j}^{k} .
$$

In particular, $\nabla_{\partial_{j}} d x^{k}=-\sum_{i} \Gamma_{i j}^{k} d x^{i}$ and

$$
\nabla d x^{k}=-\sum_{i j} \Gamma_{i j}^{k} d x^{i} \otimes d x^{j} .
$$

In general, (2.6) now implies that for a tensor $T$ of degree $(k, l)$,

$$
\begin{gathered}
T_{j_{1}, \ldots, j_{l} ; j}^{i_{1}, \ldots, i_{k}}=T_{j_{1}, \ldots, j_{l}, j}^{i_{1}, \ldots, i_{k}}+\sum_{m} T_{j_{1}, \ldots, j_{l}}^{m, i_{2}, \ldots, i_{k}} \Gamma_{m j}^{i_{1}}+\cdots+\sum_{m} T_{j_{1}, \ldots, j_{l}}^{i_{1}, \ldots, i_{k-1}, m} \Gamma_{m j}^{i_{k}} \\
-\sum_{m} T_{m, j_{2}, \ldots, j_{l}}^{i_{1}, \ldots, i_{k}} \Gamma_{j_{1} j}^{m}-\cdots-\sum_{m} T_{j_{1}, \ldots, j_{l-1}, m}^{i_{1}, \ldots, i_{k}} \Gamma_{j_{l} j}^{m} .
\end{gathered}
$$

Remark. Given a vector bundle, an operator $\nabla$ that for each vector field $X$ and section $S$ of the bundle yields a section $\nabla_{X} S$ satisfying the analogues of conditions (i)-(iii) in Theorem 2.9.1 is called an affine connection in the bundle. An affine connection on a manifold is thus the same as an affine connection in the tangent bundle, and the theorem says that it defines a natural connection in each tensor bundle.

Now consider a Riemannian manifold with a metric tensor $g$ and an arbitrary affine connection $\nabla$. If $X$ and $Y$ are two vector fields, we have by (2.6), for any vector fields $X, Y, Z$,

$$
\nabla g(Y, Z, X)=\left(\nabla_{X} g\right)(Y, Z)=X(g(Y, Z))-g\left(\nabla_{X} Y, Z\right)-g\left(Y, \nabla_{X} Z\right) \text {. }
$$

The condition on the Riemannian (Levi-Civita) connection that $X(g(Y, Z))=$ $g\left(\nabla_{X} Y, Z\right)+g\left(Y, \nabla_{X} Z\right)$ can thus be written as $\nabla g=0$. In other words, the Riemannian connection is characterized as the unique affine connection that is symmetric (cf. Example 2.7.5) and is such that $\nabla g=0$.

2.10. Tensors on a Riemannian manifold. Suppose that $M$ is a Riemannian manifold, with metric tensor $g$ and Riemannian (Levi-Civita) connection $\nabla$. Recall from Section 2.9 that $\nabla g=0$.

We can raise or lower indices of tensors on $M$ using the Riemannian metric $g$, by applying the isomorphisms described in Section 1.9 at each point $p$; recall that the Riemannian metric by definition makes each $T_{p}(M)$ into a Euclidean space. Thus there

Example 2.10.1. The curvature tensor $R$ has a covariant form (also denoted by $R$ ), which is a covariant tensor of degree 4 . The connection with the tensor of degree $(1,3)$ defined in Example 2.7.6 is

$$
R(X, Y, Z, W)=\langle R(X, Y) Z, W\rangle,
$$

for arbitrary vector fields (or just tangent vectors at a single point) $X, Y, Z, W$. 
Example 2.10.2. For the coefficients in a coordinate system let, as usual, $g^{i j}$ be the coefficients of the dual inner product so that $\left(g^{i j}\right)$ is the matrix inverse of $\left(g_{i j}\right)$. If $R_{i j k}^{l}$ and $R_{i j k l}$ denote two forms of the Riemann curvature tensor, $R_{i j}$ the Ricci tensor and $R$ the scalar curvature obtained by contracting the Ricci tensor, we have

$$
\begin{aligned}
R_{i j k}^{l} & =\sum_{m} g^{l m} R_{i j k m} \\
R_{i j k l} & =\sum_{m} g_{l m} R_{i j k}^{m} \\
R_{i j} & =\sum_{k} R_{i k j}^{k}=\sum_{k, l} g^{k l} R_{i k j l}=\sum_{k, l} g^{k l} R_{k i l j} \\
R & =\sum_{i, j} g^{i j} R_{i j}=\sum_{i, j, k, l} g^{i k} g^{j l} R_{i j k l} .
\end{aligned}
$$

If $X$ is a vector field and $T$ is any tensor field, then $\nabla_{X}(g \otimes T)=\left(\nabla_{X} g\right) \otimes$ $T+g \otimes \nabla_{X} T=g \otimes \nabla_{X} T$ by Theorem 2.9.1(vi). Since lowering an index of $T$ can be described as taking the tensor product with $g$ followed by a contraction (Section 1.9), and $\nabla_{X}$ commutes with contractions, it follows that $\nabla_{X}$ commutes with lowerings of indices, and thus also with raisings of indices.

Example 2.10.3. The covariant form of the Riemann curvature tensor has coefficients $R_{i j k l}$ in a coordinate system, and its covariant differential $\nabla R$ has coefficients $R_{i j k l ; m}$. The Bianchi identities are

$$
R_{i j k l}+R_{i k l j}+R_{i l j k}=0
$$

and

$$
R_{i j k l ; m}+R_{i j l m ; k}+R_{i j m k ; l}=0 .
$$

Raising the indices $i$ and $j$ in the second Bianchi identity and contracting the two pairs $(i, k)$ and $(j, l)$, we obtain, since covariant differentiation commutes with contractions and raisings of indices,

$$
R_{; m}-\sum_{i} R_{m ; i}^{i}-\sum_{l} R_{m ; l}^{l}=0
$$

where $R_{j}^{i}$ denotes the coefficients of (the mixed form of) the Ricci tensor, and $R=\sum_{i} R_{i}^{i}$ is the scalar curvature. It follows that if $E^{i}{ }_{j}=R^{i}{ }_{j}-\frac{1}{2} \delta_{j}^{i} R$ denotes the coefficients of the mixed form of the Einstein tensor, then, using the easily verified fact that the tensor $\iota$ with coefficients $\delta_{j}^{i}$ has $\nabla \iota=0$ (cf. Example 1.9.2),

$$
\sum_{i} E_{j ; i}^{i}=0
$$

(The covariant form of the Einstein tensor is $E_{i j}=R_{i j}-\frac{1}{2} R g_{i j}$.) 


\section{Differential FORMS}

A differential form of degree $k$, often simply called a $k$-form, on a manifold is an antisymmetric covariant tensor field (of degree $k$ ). More generally, we can define a differential form as a (formal) sum $\omega_{0}+\omega_{1}+\cdots+\omega_{n}$, where $\omega_{k}$ is a differential form of degree $k$. The value at a point $p$ of a differential form is thus an element of the exterior algebra $A\left(T_{p}(M)\right)$; if the differential form has degree $k$, then the value belongs to the subspace $A_{k}\left(T_{p}(M)\right)$.

By Theorem 2.7.1, a $k$-form may be regarded as an antisymmetric $C^{\infty}$. multilinear mapping $\omega: \mathcal{X}(M)^{k} \rightarrow C^{\infty}(M)$.

We let $E^{k}(M)$ denote the linear space of all $k$-forms on $M$, and $E^{*}(M)=$ $\bigoplus_{k=0}^{n} E^{k}(M)$ the linear space of all differential forms.

Remark. Using the theory of vector bundles, we may form the exterior bundle $A(M)=\bigcup_{p \in M} A\left(T_{p}(M)\right)$ and its subbundles $A_{k}(M)=\bigcup_{p \in M} A_{k}\left(T_{p}(M)\right), 0 \leq$ $k \leq n$. A differential form (of degree $k$ ) is then a section in the exterior bundle (in $A_{k}(M)$ ).

We form the sum and exterior product of two differential forms by performing the operations pointwise; thus the space $E^{*}(M)$ of all differential forms becomes an algebra. This algebra is associative but not commutative; it is by (1.7) anticommutative in the sense that

$$
\omega_{2} \wedge \omega_{1}=(-1)^{k l} \omega_{1} \wedge \omega_{2}, \quad \omega_{1} \in E^{k}(M), \omega_{2} \in E^{l}(M) .
$$

3.1. Bases. Given a frame $\left\{E_{1}, \ldots, E_{n}\right\}$ in an open subset $U$ of $M$, the dual frame $\left\{E^{1}, \ldots, E^{n}\right\}$ gives a basis in $T_{q}^{*}(M)$ for every $q \in U$, and by taking exterior products, we obtain a basis in each $A_{k}\left(T_{p}(M)\right)$.

In particular, if $\left(x^{1}, \ldots, x^{n}\right)$ is a coordinate system in an open set $U$, the differentials $d x^{1}, \ldots, d x^{n}$ form a basis in each $T_{q}^{*}(M)$, and the exterior products $d x^{i_{1}} \wedge \ldots d x^{i_{k}}, i_{1}<\cdots<i_{k}$, form a collection of differential forms on $U$ that yield a basis in $A_{k}\left(T_{q}(M)\right)$ for every $q \in M(0 \leq k \leq n)$. Hence, any differential form on $U$ may uniquely be written

$$
\sum_{k=0}^{n} \sum_{1 \leq i_{1}<\ldots i_{k} \leq n} a_{i_{1}, \ldots, i_{k}} d x^{i_{1}} \wedge \cdots \wedge d x^{i_{k}}
$$

for some smooth functions $a_{i_{1}, \ldots, i_{k}}$ on $U$.

3.2. Exterior differentiation. If $f$ is a smooth function on $M$, i.e. a 0 -form, its differential $d f$ was defined in Section 2 as a cotangent field, i.e. a 1-form (Section 2.1 and Example 2.4.5). This operation $d$ has an important extension to differential forms described by the following theorem; $d$ is called exterior differentiation.

Theorem 3.2.1. There exists a unique operator $d: E^{*}(M) \rightarrow E^{*}(M)$ with the following properties:

(i) $d: E^{*}(M) \rightarrow E^{*}(M)$ is linear.

(ii) If $\omega \in E^{k}(M)$, then $d \omega \in E^{k+1}(M)$. 
(iii) If $\omega_{1} \in E^{k}(M)$ and $\omega_{2} \in E^{l}(M)$, then

$$
d\left(\omega_{1} \wedge \omega_{2}\right)=\left(d \omega_{1}\right) \wedge \omega_{2}+(-1)^{k} \omega_{1} \wedge\left(d \omega_{2}\right) .
$$

(iv) $d \circ d=0$, i.e. $d(d \omega)=0$ for every $\omega \in E^{*}(M)$.

(v) If $f \in E^{0}(M)=C^{\infty}(M)$, then $d f \in E^{1}(M)$ is the differential of $f$ given by $d f(X)=X f, X \in \mathcal{X}(M)$.

Moreover, $d$ is a local operator in the sense that for every $p \in M, d \omega(p)$ depends only on the values of $\omega$ in a neighbourhood of $p$ (i.e. on the germ of $\omega$ at $p)$. Explicitly, in a coordinate system, $d$ is given by

$$
\begin{aligned}
d\left(\sum a_{i_{1}, \ldots, i_{k}} d x^{i_{1}} \wedge \cdots \wedge d x^{i_{k}}\right) & =\sum d a_{i_{1}, \ldots, i_{k}} d x^{i_{1}} \wedge \cdots \wedge d x^{i_{k}} \\
& =\sum \frac{\partial a_{i_{1}, \ldots, i_{k}}}{\partial x^{j}} d x^{j} \wedge d x^{i_{1}} \wedge \cdots \wedge d x^{i_{k}} .
\end{aligned}
$$

Proof. Suppose first that $M$ is covered by a single coordinate neighbourhood, and let $\left(x^{1}, \ldots, x^{n}\right)$ be a global coordinate system. Then every differential form on $M$ can be written as (3.2) for some smooth functions $a_{i_{1}, \ldots, i_{k}}$. If $d$ satifies the conditions, then (3.3) follows directly, which further shows that $d$ is local and unique. Conversely, we may define $d$ by (3.3), and it is easily verified that (i) - (v) hold. (For (iv), first note that if $f$ is a smooth function, then

$$
d d f=d\left(\sum_{i} \frac{\partial f}{\partial x^{i}} d x^{i}\right)=\sum_{i, j} \frac{\partial^{2} f}{\partial x^{i} \partial x^{j}} d x^{j} \wedge d x^{i}=0,
$$

because $\frac{\partial^{2} f}{\partial x^{i} \partial x^{j}}=\frac{\partial^{2} f}{\partial x^{j} \partial x^{i}}$ and $d x^{j} \wedge d x^{i}=-d x^{i} \wedge d x^{j}$. The general case then follows by (3.3).)

For a general manifold $M$, suppose that $d$ satisfies (i)-(v). First, assume that $p \in M$ and that $\omega_{1}, \omega_{2} \in E^{*}(M)$ coincide in a neighbourhood $V$ of $p$. Let $\omega=\omega_{1}-\omega_{2}$ and choose $\varphi \in C^{\infty}(M)$ such that $\varphi(p)=1$ and $\operatorname{supp} \varphi \subset V$. Then $\varphi \omega=0$ and $d \varphi \wedge \omega=0$, and since (iii) yields $d(\varphi \omega)=d \varphi \wedge \omega+\varphi \wedge d \omega$, we obtain $\varphi d \omega=\varphi \wedge d \omega=0$. In particular $d \omega(p)=0$ and thus $d \omega_{1}(p)=d \omega_{2}(p)$. This shows that $d$ is a local operator.

Next, let $U$ be an open subset of $M$. If $\omega \in E^{*}(U)$ and $p \in U$, we can find $\bar{\omega} \in E^{*}(M)$ such that $\bar{\omega}=\omega$ in a neighbourhood of $p$. By the fact just shown that $d$ is local, $d \bar{\omega}(p)$ does not depend on the choice of $\bar{\omega}$, so we may uniquely define $d_{U} \omega(p)=d \bar{\omega}(p)$. It is easily seen that $d_{U} \omega \in E^{*}(U)$ and that we have defined an operator $d_{U}: E^{*}(U) \rightarrow E^{*}(U)$ that satisfies (i)-(v) on $U$. In particular, if $U$ is a coordinate neighbourhood, the first part of the proof applies and shows that $d_{U}$ is given by (3.3). Moreover, if $\omega \in E^{*}(M)$ and $\omega_{U}$ is the restriction of $\omega$ to $U$, we can choose $\bar{\omega}_{U}=\omega$ which shows that $d \omega=d_{U} \omega_{U}$ on $U$. Consequently, $d \omega$ is given by (3.3) on $U$. This further shows uniqueness in the general case.

For existence, finally, we can for any $\omega \in E^{*}(M)$ use (3.3) to define $d \omega$ in any coordinate neighbourhood. We must, however, verify that these definitions are consistent. Thus, suppose that $U$ and $\bar{U}$ are two open sets where coordinate systems $\left(x^{1}, \ldots, x^{n}\right)$ and $\left(\bar{x}^{1}, \ldots, \bar{x}^{n}\right)$, respectively, are defined, and define the corresponding operators $d_{U}$ on $E^{*}(U)$ and $d_{\bar{U}}$ on $E^{*}(\bar{U})$ by $(3.3)$, using the 
respective coordinate systems. On the intersection $U \cap \bar{U}$, both coordinate systems are defined and thus define differentiation operators in $E^{*}(U \cap \bar{U})$ by (3.3); by the uniqueness shown in the first part of the proof, these operators coincide, and thus $d_{U} \omega=d_{\bar{U}} \omega$ on $U \cap \bar{U}$ for any $\omega \in E^{*}(M)$, which is the requied consistency. Hence, $d \omega$ is well defined, and it is easily checked that (i)-(v) hold.

Remark. The sign $(-1)^{k}$ in (iii) may look strange, but it is easily verified that it is consistent with (and essentially forced by) the anticommutation rule (3.1).

Exterior differentiation can also be described by coordinate-free explicit formulas.

Theorem 3.2.2. Regarding differential forms as alternating multilinear operators on $\mathcal{X}(M)$, we have for arbitrary vector fields $X, Y, X_{1}, \ldots$

$$
\begin{array}{rlrl}
d f(X) & =X f, \quad f \in C^{\infty}(M) & =E^{0}(M) ; \\
d \omega(X, Y) & =X(\omega(Y))-Y(\omega(X))-\omega([X, Y]), & \omega & \in E^{1}(M) ;
\end{array}
$$

and more generally, for $\omega \in E^{k}(M)$,

$$
\begin{aligned}
d \omega\left(X_{1}, \ldots, X_{k+1}\right)= & \sum_{i=1}^{k+1}(-1)^{i-1} X_{i}\left(\omega\left(X_{1}, \ldots, \hat{X}_{i}, \ldots, X_{k+1}\right)\right) \\
& +\sum_{i<j}(-1)^{i+j}\left(\omega\left(\left[X_{i}, X_{j}\right], X_{1}, \ldots, \hat{X}_{i}, \ldots, \hat{X}_{j}, \ldots, X_{k+1}\right)\right),
\end{aligned}
$$

where $\hat{X}_{i}$ denotes omitted terms.

Proof. First, (3.4) is the same as Theorem 3.2.1(v).

For (3.5), let us denote the right hand side by $d^{\prime} \omega(X, Y)$. Consider first the case when $\omega=d f$ for some $f \in C^{\infty}(M)$. Then $d^{\prime} \omega(X, Y)$ equals, by (3.4), $X(Y f)-Y(X f)-[X, Y] f$, which vanishes by the definition of $[X, Y]$; since $d \omega=d d f=0,(3.5)$ holds in this case.

Next, for any smooth function $f$ and 1 -form $\omega$, it is easily seen that

$$
\begin{aligned}
d^{\prime}(f \omega)(X, & Y)-f d^{\prime} \omega(X, Y) \\
& =X(f \omega(Y))-f X(\omega(Y))-Y(f \omega(X))+f Y(\omega(X)) \\
& =X(f) \omega(Y)-Y(f) \omega(X)=d f(X) \omega(Y)-d f(Y) \omega(X) \\
& =(d f \wedge \omega)(X, Y) .
\end{aligned}
$$

Thus $d^{\prime}(f \omega)-f d^{\prime} \omega=d f \wedge \omega=d(f \omega)-f d \omega$, so if further $d^{\prime} \omega=d \omega$, then $d^{\prime}(f \omega)=d(f \omega)$.

Combining these two steps, and the fact that any 1-form $\omega$ locally is a linear combination $\sum f_{j} d x^{j}$, we see that $d^{\prime} \omega=d \omega$.

The proof of (3.6) is similar (but notationally more complicated), and is omitted. 
3.3. Closed and exact forms. A differential form $\omega$ is closed if $d \omega=0$, and exact if $\omega=d \tilde{\omega}$ for some differential form $\tilde{\omega}$, i.e. if $\omega \in \operatorname{Im}(d)$.

Since $d \circ d=0$, every exact form is closed. For $k$-forms with $k \geq 1$, the converse is true locally, for example in any open set diffeomorphic to an open ball in $\mathbb{R}^{n}$ (the Poincaré lemma, see $[1,3]$ for proofs), but not always globally. (The case of 0 -forms is trivial: there are no exact 0 -forms except 0 , while every constant function is a closed 0 -form; in a connected manifold the constants are furthermore the only closed 0 -forms.)

Example 3.3.1. Let $M=\mathbb{R}^{2} \backslash\{0\}$. Then $\omega=(x d y-y d x) /\left(x^{2}+y^{2}\right)$ is a closed 1-form that is not exact. This can be verified by direct computations, but it is more illuminating to observe that in any subset of $M$ where we can define a continuous branch of $\arg (x+i y)=\operatorname{Im} \log (x+i y)$, we have $\omega=d \arg$. This shows that $\omega$ is closed (locally and thus globally); it also shows that if $\omega=d f$ for some function $f$, then $f$ would have to be a constant plus a globally defined continuous branch of arg, which is impossible.

The de Rham cohomology groups are defined as the quotient spaces

$$
\left.H^{k}(M)=\{\text { closed } k \text {-forms }\} / \text { exact } k \text {-forms }\right\} \text {. }
$$

Thus $H^{k}(M)=0$ if and only if every closed $k$-form is exact.

It turns out that the cohomology groups are finite-dimensional in many cases, for example whenever $M$ is compact. In a vague sense, the dimension of $H^{k}(M)$ (known as the Betti number) is the number of $k$-dimensional "holes" in $M$.

Remark. For any manifold $M$, the de Rham cohomology groups are isomorphic to the singular and Čech cohomology groups (with real coefficients) defined in algebraic topology, see [3].

Example 3.3.2. $H^{k}\left(R^{n}\right)=0$ for every $k \geq 1$.

$H^{k}\left(S^{n}\right)=0$ for $1 \leq k<n$ (and trivially for $k>n$ ), while $H^{n}\left(S^{n}\right) \cong \mathbb{R}$.

3.4. Classical vector analysis. Classically, a vector field on $\mathbb{R}^{3}$ (or on an open subset) is a (smooth) function into $\mathbb{R}^{3}$, or equivalently a triple $\left(f_{1}, f_{2}, f_{3}\right)$ of smooth real-valued functions. This can be identified with the vector field (in general differential geometry sense) $f_{1} \partial / \partial x^{1}+f_{2} \partial / \partial x^{2}+f_{3} \partial / \partial x^{3}$.

We can also identify a 1 -form $f_{1} d x^{1}+f_{2} d x^{2}+f_{3} d x^{3}$ with the vector field $\left(f_{1}, f_{2}, f_{3}\right)$; this amounts to identifying a 1 -form and the vector field obtained by raising the index as in Sections 1.9 and 2.10.

Moreover, a 2-form may be written $f_{12} d x^{1} \wedge d x^{2}+f_{13} d x^{1} \wedge d x^{3}+f_{23} d x^{2} \wedge$ $d x^{3}$, and we may identify this with the vector field $\left(f_{23},-f_{13}, f_{12}\right)$; the order and signs of the functions are chosen such that if $\omega$ is a 2 -form and $F$ the corresponding vector field, and $\lambda$ is any 1-form, then

$$
\lambda \wedge \omega=\langle\lambda, F\rangle d x^{1} \wedge d x^{2} \wedge d x^{3} .
$$

Finally, a 3-form can be written $f d x^{1} \wedge d x^{2} \wedge d x^{3}$ and identified with the function $f$. 
With these identifications, the exterior differentiation $d$ yields three classical operators: $d: E^{0}\left(\mathbb{R}^{3}\right) \rightarrow E^{1}\left(\mathbb{R}^{3}\right)$ becomes the gradient grad : $C^{\infty} \rightarrow \mathcal{X}$, $d: E^{1}\left(\mathbb{R}^{3}\right) \rightarrow E^{2}\left(\mathbb{R}^{3}\right)$ becomes the curl $: \mathcal{X} \rightarrow \mathcal{X}$, and $d: E^{2}\left(\mathbb{R}^{3}\right) \rightarrow E^{3}\left(\mathbb{R}^{3}\right)$ becomes the divergence div : $\mathcal{X} \rightarrow C^{\infty}$.

The fact that $d \circ d=0$ thus says that the gradient of a function is curl-free, and that the curl of a vector field is divergence-free. Conversely, the Poincaré lemma says that (globally or in a suitable, for example convex, subset), every curl-free vector field is the gradient of some function, and that every divergencefree vector field is the curl of another vector field.

3.5. Gradient and divergence on Riemannian manifolds. While the curl studied in Section 3.4 is a special phenomenon in three dimensions, the gradient and divergence may be defined in any Riemannian manifold $M$. First, if $f \in$ $C^{\infty}(M)$, then $d f$ is a 1 -form, and we define grad $f$ as the corresponding vector field, obtained by raising the index.

Secondly, if $X$ is a vector field, its covariant derivative $\nabla X$ is a tensor of degree $(1,1)$, and we define $\operatorname{div} X$ to be the contraction of $\nabla X$. Thus $\operatorname{grad}: C^{\infty}(M) \rightarrow \mathcal{X}(M)$ and $\operatorname{div}: \mathcal{X}(M) \rightarrow C^{\infty}(M)$.

The composition div grad : $C^{\infty}(M) \rightarrow C^{\infty}(M)$ is known as the LaplaceBeltrami operator $\Delta$. Equivalently, $\Delta f$ is the contraction of the second covariant derivative $\nabla \nabla f \in T^{0,2}(M)$, and in a coordinate system we have

$$
\Delta f=\sum_{i, j} g^{i j} f_{; i j}
$$

Remark. The divergence can also be described using an isomorphism between vector fields and $(n-1)$-forms as in the case of $\mathbb{R}^{3}$ studied above. More generally, if $M$ is oriented (otherwise we can work locally), there exists a unique $n$-form $\omega_{0}$ such that if $\left(x^{1}, \ldots, x^{n}\right)$ is any oriented coordinate system, then

$$
\omega_{0}=\left(\operatorname{det}\left(g_{i j}\right)_{i j}\right)^{1 / 2} d x^{1} \wedge \cdots \wedge d x^{n} .
$$

There are now isomorphisms $C^{\infty}(M) \cong E^{n}(M)$ and $\mathcal{X}(M) \cong E^{n-1}(M)$ such that a function $f$ corresponds to the $n$-form $f \omega_{0}$, and a vector field $X$ to the unique $(n-1)$-form $\omega$ such that $\lambda \wedge \omega=\langle\lambda, X\rangle \omega_{0}$ for every 1-form $\lambda$. It may then be verified that $d: E^{n-1}(M) \rightarrow E^{n}(M)$ corresponds to $\operatorname{div}: \mathcal{X}(M) \rightarrow$ $C^{\infty}(M)$.

3.6. Pull-backs. Suppose that $\varphi: M \rightarrow N$ is a smooth mapping between two manifolds. If $f: N \rightarrow \mathbb{R}$ is a smooth function on $N$, then $f \circ \varphi$ is a smooth function on $M$. This operation extends in a natural way to differential forms.

Theorem 3.6.1. There is a unique linear map $\varphi_{*}: E^{*}(N) \rightarrow E^{*}(M)$ such that $\varphi_{*}(f)=f \circ \varphi$ for $f \in C^{\infty}(M)=E^{0}(N), \varphi_{*}(d \omega)=d\left(\varphi_{X}(\omega)\right)$ and $\varphi_{*}\left(\omega_{1} \wedge \omega_{2}\right)=$ $\left(\varphi_{*} \omega_{1}\right) \wedge\left(\varphi_{*} \omega_{2}\right)$ for arbitrary differential forms $\omega, \omega_{1}, \omega_{2}$.

The operator $\varphi_{*}$ is known as the pull-back. 


\section{INTEGRATION OF DIFFERENTIAL FORMS}

Consider an $n$-form $\omega$ on the $n$-dimensional manifold $M$. If $\mathbf{x}=\left(x^{1}, \ldots, x^{n}\right)$ is a coordinate system in an open set $U$, then $\omega=f d x^{1} \wedge \cdots \wedge d x^{n}$ in $U$ for some smooth function $f$.

If $\mathbf{y}=\left(y^{1}, \ldots, y^{n}\right)$ is another coordinate system, defined in an open set $U^{\prime}$, say, then we also have $\omega=g d y^{1} \wedge \cdots \wedge d y^{n}$ in $U^{\prime}$. On the intersection $U \cap U^{\prime}$, $d y^{i}=\sum_{j} \frac{\partial y^{i}}{\partial x^{j}} d x^{j}$ and thus, by (1.9),

$$
d y^{1} \wedge \cdots \wedge d y^{n}=J d x^{1} \wedge \cdots \wedge d x^{n}
$$

where $J=\operatorname{det}\left(\left(\partial y^{i} / \partial x^{j}\right)_{i, j=1}^{n}\right)$ is the Jacobian of the change of coordinates $\mathbf{y} \circ \mathbf{x}^{-1}$. Consequently, $\omega=g d y^{1} \wedge \cdots \wedge d y^{n}=g J d x^{1} \wedge \cdots \wedge d x^{n}$, and thus $f=g J$.

We here regard $f, g$ and $J$ as functions defined on (subsets of) $M$; the coordinate representations are the functions $\bar{f}=f \circ \mathbf{x}^{-1}$ defined on $\mathbf{x}(U)$, $\bar{g}=g \circ \mathbf{y}^{-1}$ defined on $\mathbf{y}\left(U^{\prime}\right)$, and $\bar{J}=J \circ \mathbf{x}^{-1}$ defined on $\mathbf{x}\left(U \cap U^{\prime}\right)$ (all three are open subsets of $\mathbb{R}^{n}$ ).

Next, let $K$ be a compact (for convenience) subset of $U \cap U^{\prime}$. By the standard formula for a change of variables in a multidimensional integral,

$$
\begin{aligned}
\int_{\mathbf{y}(K)} \bar{g} d y^{1} \cdots d y^{n} & =\int_{\mathbf{x}(K)} \bar{g} \circ\left(\mathbf{y} \circ \mathbf{x}^{-1}\right)|\bar{J}| d x^{1} \cdots d x^{n} \\
& =\int_{\mathbf{x}(K)} g \circ \mathbf{x}^{-1}\left|J \circ \mathbf{x}^{-1}\right| d x^{1} \cdots d x^{n} \\
& =\int_{\mathbf{x}(K)} f \circ \mathbf{x}^{-1} \operatorname{sgn}\left(J \circ \mathbf{x}^{-1}\right) d x^{1} \cdots d x^{n} \\
& =\int_{\mathbf{x}(K)} \bar{f} \operatorname{sgn}(\bar{J}) d x^{1} \cdots d x^{n} .
\end{aligned}
$$

Consequently, if the two coordinate systems $\mathbf{x}$ and $\mathbf{y}$ have the same orientation, i.e. $J>0$, then $\int_{\mathbf{y}(K)} \bar{g}=\int_{\mathbf{x}(K)} \bar{f}$, while the two integrals differ by sign if the two coordinate systems have different orientations.

From now on, suppose that $M$ is an oriented manifold. The argument just given shows that if $\omega$ is an $n$-form and $K$ is a compact subset of $M$ that is covered by a single coordinate neighbourhood, then we may uniquely define

$$
\int_{K} \omega=\int_{\mathbf{x}(K)} \bar{f} d x^{1} \cdots d x^{n}
$$

for any positively oriented coordinate system $\mathbf{x}=\left\{e^{1}, \ldots, e^{n}\right\}$ defined on $K$, with $\bar{f}$ as above. A routine argument using partitions of $K$ or of $\omega$ then shows that we may (in a natural way, preserving linearity properties) define $\int_{K} \omega$ for any $n$-form $\omega$ and any compact subset $K$ of $M$, and $\int_{M} \omega$ for any $n$-form $\omega$ with compact support. The compactness conditions may be relaxed to an integrability condition.

Remark. Note that only $n$-forms can be integrated over an $n$-dimensional manifold. However, forms of lower degree can be integrated over submanifolds of 
the corresponding dimension. Thus, if $\omega$ is a $k$-form and $N$ is a $k$-dimensional submanifold of $M$, then the restriction (i.e. the pull-back) of $\omega$ to $N$ is a $k$-form on $N$ and $\int_{N} \omega$ is defined provided, for example, $\omega$ has compact support in $N$.

Similarly, if $\alpha:(a, b) \rightarrow M$ is a (smooth) curve and $\omega$ is a 1-form, the integral $\int_{\alpha} \omega$ is defined (under suitable integrability restrictions) by integrating the pull-back of $\omega$ to $(a, b)$; it is easily verified that this integral is independent of the parametrization of $\alpha$. Thus line integrals of 1 -forms are defined very generally.

4.1. Integration on Riemannian manifolds. Every Riemannian manifold (oriented or not) carries a natural measure, which in a coordinate system $\left(x^{1}, \ldots, x^{n}\right)$ is given by

$$
d \mu=\sqrt{\operatorname{det}\left(\left(g_{i j}\right)_{i j}\right)} d x^{1} \cdots d x^{n} .
$$

(This equals the $n$-dimensional Hausdorff measure defined by the metric.)

Thus we may integrate functions on a Riemannian manifold. If $M$ is oriented, and $\omega_{0}$ is the special $n$-form defined (3.8), then $\int_{M} f=\int_{M} f \omega_{0}$, so the two notions of integration correspond in a natural way.

4.2. Stokes' theorem. Let $M$ be an oriented manifold. A regular domain in $M$ is a subset of the form $\{p \in M: f(p)>0\}$ for some smooth function $f: M \rightarrow \mathbb{R}$ such that $d f \neq 0$ at every point $p$ with $f(p)=0$; equivalently (as follows by a partition of unity argument), $D$ is an open subset such that every boundary point $p \in \partial U$ has a neighbourhood $U$ with a smooth function $f: U \rightarrow \mathbb{R}$ such that $d f \neq 0$ in $U$ and $D \cap U=\{p \in U: f(p)>0\}$.

If $D$ is a regular domain, then its boundary $\partial D$ is an $(n-1)$-dimensional manifold, embedded in $M$. The orientation of $M$ induces an orientation of $\partial D$; we choose the orientation such that if $p \in \partial D$ and $v \in T_{p}(M) \backslash T_{p}(\partial D)$ is an outer tangent vector, meaning that $v=\alpha^{\prime}(0)$ for some curve $\alpha$ with $\alpha(0)=p$ and $\alpha(t) \notin M$ for $t>0$, then $v_{1}, \ldots, v_{n-1}$ is an oriented basis in $T_{p}(\partial D)$ if and only if $v, v_{1}, \ldots, v_{n-1}$ is an oriented basis in $T_{p}(M)$. Then Stoke's theorem may be stated as follows.

Theorem 4.2.1 (Stokes). Let $D$ be a regular domain in an oriented manifold $M$ of dimension $n$, and let $\partial D$ have the induced orientation. If $\omega$ is an $(n-1)-$ form on $M$, and either $\bar{D}$ or $\operatorname{supp} \omega$ is compact, then

$$
\int_{D} d \omega=\int_{\partial D} \omega
$$

Proof. Using a partition of unity and suitable coordinate mappings, the theorem is reduced to the case where $D$ is the upper half space in $M=\mathbb{R}^{n}$ and $\omega$ has compact support, which is verified by a simple calculation.

Corollary 4.2.1. If $M$ is an oriented n-dimensional manifold and $\omega$ is an $(n-1)$-form on $M$ with compact support, then

$$
\int_{M} d \omega=0
$$


Remark. An essentially equivalent, but sometimes more elegant version of Stokes' theorem uses the notion of manifolds with boundary instead of regular domains, see [1].

This general version of Stokes' theorem contains several standard results from calculus.

Example 4.2.1. If $D$ is a bounded regular domain in $\mathbb{R}^{2}$ and $\omega=f d x+g d y$ is a 1-form, then Stokes' theorem yields

$$
\int_{D}\left(\frac{\partial g}{\partial x}-\frac{\partial f}{\partial y}\right) d x \wedge d y=\int_{\partial D} f d x+g d y
$$

where the left hand side is an ordinary Riemann integral (we may replace $d x \wedge d y$ by $d x d y$ ) and the right hand side is a line integral. This is known as Green's theorem.

Example 4.2.2. Let $M$ be a smooth surface in $\mathbb{R}^{3}$, and $D$ a regular domain in $M$, i.e. a portion of the surface bounded by smooth curves. Denote the unit normal to $M$ (suitably oriented) by $n$. Let $\omega=f d x+g d y+h d z$ be a 1 -form in $\mathbb{R}^{3}$, and let $F=(f, g, h)$ denote the corresponding vector field as in Section 3.4. If $\omega_{0}$ is the special 2-form on $M$ defined by (3.8) and $\tilde{\omega}_{0}=d x \wedge d y \wedge d z$ is the corresponding special 3-form on $\mathbb{R}^{3}$, then $\tilde{\omega}_{0}=n \wedge \omega_{0}$ on $M$. Thus, if $d \omega=\varphi \omega_{0}$, we have $n \wedge d \omega=\varphi \tilde{\omega}_{0}$. On the other hand, by Section 3.4, in particular (3.7), $n \wedge d \omega=\langle n, \operatorname{curl} F\rangle \tilde{\omega}_{0}$; hence $\varphi=\langle n, \operatorname{curl} F\rangle$. Consequently, Stokes' theorem yields

$$
\int_{\partial D} f d x+g d y+h d z=\int_{D} d \omega=\int_{D}\langle n, \operatorname{curl} F\rangle
$$

where the final integral is an ordinary integral with respect to surface measure, cf. Section 4.1 .

Example 4.2.3. In a Riemannian manifold, for example in $\mathbb{R}^{n}$, the isomorphisms in the remark in Section 3.5 yield the following version of Stokes' theorem, known as the divergence theorem.

Theorem 4.2.2. Let $D$ be a regular domain in a Riemannian manifold $M$, and let, for $p \in \partial D, n(p)$ be the outer unit normal to $D$. If $X$ is a vector field on $M$, and either $\bar{D}$ or $\operatorname{supp} X$ is compact, then

$$
\int_{D} \operatorname{div} X=\int_{\partial D}\langle X, n\rangle
$$

Here both integrals are ordinary (Lebesgue) integrals with respect to the standard measure (4.1) in a Riemannian manifold.

\section{REFERENCES}

[1] W. Boothby, An Introduction to Differentiable Manifolds and Riemannian Geometry. Academic Press, New York, 1975.

[2] M. do Carmo, Riemannian Geometry. Birkhäuser, Boston, 1992.

[3] F. Warner, Foundations of Differentiable Manifolds and Lie Groups. Springer, New York, 1983. 
Department of Mathematics, Uppsala University, PO Box 480, S-751 06 UppSALA, SWEDEN

E-mail address: svante.janson@math.uu.se 